\section{Christoph Bachmann}

\section{Einleitung}

Bei Diskussionen über Homöopathie steht meistens das Bemühen im Zentrum, das richtige homöopathische Medikament, das sogenannte Simile, zu finden. Dieses kann einen Patienten nicht nur von gegenwärtigen Symptomen einer Krankheit befreien, sondern in vielen Fällen das Rezidivieren einer Krankheit verhindern. Dies ist das Ziel homöopathisch tätiger Ärzte und Therapeuten und kann bei den von lang anhaltenden Krankheiten geplagten und nun davon befreiten Patienten ungläubige Freude auslösen. Denn: Oft wurden diese von verschiedenen schulmedizinisch tätigen Ärzten erfolglos behandelt und sind nun auf einmal nachhaltig beschwerdefrei! Möglich wird dies - leider natürlich nicht in jedem Fall! - durch sorgfältige Fallaufnahme, differenzierte Klärung spezieller Umstände und achtsame Beobachtung des Patienten und anschliessend genauer Repertorisation. Dann folgt ein Vergleich der infrage kommenden homöopathischen Arzneimittel in der Materia medica, was schliesslich zur Auswahl eines homöopathischen Arzneimittels in der geeigneten Potenz führt.

\section{Herstellung}

Bei den meisten homöopathischen Fortbildungen wird über neue Methoden von Anamnese, Befragungen, Repertorisierung und Vergleich der Materia medica gesprochen. Manchmal kommt bei solchen Anlässen auch die Herstellung der homöopathischen Arzneimittel zur Sprache, was ein nicht zu unterschätzender Aspekt in

\title{
Herstellung und Abgabe homöopathischer Arzneimittel in der Schweiz: Rechtliche Bestimmungen und Umsetzung
}

Im Zusammenhang mit homöopathischen Behandlungen durch ärztliche Homöopathen oder Heilpraktiker gibt es für die Herstellung und Abgabe homöopathischer Arzneimittel eine Reihe von rechtlichen Bestimmungen, über die selten gesprochen wird. Die Einhaltung dieser Vorschriften, speziell bei der Abgabe von Einzeldosen, gehört jedoch auch zu einer qualitativ hochstehenden Homöopathie.

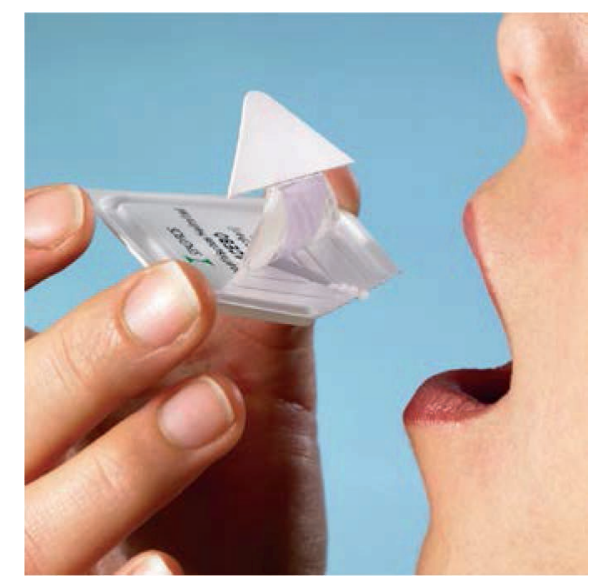

Abb. 1. Einnahme einer homöopathischen Einzeldosis.

der homöopathischen Behandlung ist. Denn was nützt eine sorgfältige Auswahl des geeigneten homöopathischen Arzneimittels, wenn der Patient schliesslich Globuli oder Tropfen einnimmt, die wegen unsorgfältiger Herstellung nicht oder zu wenig wirksam sind?

Der Frage nach einer qualitativ einwandfreien Herstellung homöopathischer Arzneimittel bzw. der Auswahl eines Herstellers solcher Präparate, der sich genau an die Vorschriften der Behörden und der auf Hahnemann zurückgehenden homöopathischen Tradition hält, sollte daher mehr Gewicht beigemessen werden.

\section{Qualität der Herstellung}

Eigentlich ist dies völlig klar: Besonders in der Homöopathie sollten behandelnde Ärzte und Therapeuten grossen Wert auf die Vertrauenswürdigkeit eines Herstellers von Arzneimitteln setzen. Verwendet eine Firma, die homöopathische Arzneimittel herstellt, genau die Ausgangsstoffe, die verwendet wurden, als die Pioniere der Homöopathie die homöopathischen Arzneimittelprüfungen vornahmen? Wie wird in solchen Firmen trituriert und potenziert?

Es ist eine Tatsache, dass homöopathische Arzneimittel, die nicht bis mindestens zur 30. Potenz, idealerweise auch höher, von Hand potenziert werden, weniger wirksam sind als diejenigen, bei der eine Person mit viel Geduld vor dem Lederbock sitzt, auf dem von Hand potenziert wird. In der Schweiz gibt es aber homöopathische Firmen, die ausschliesslich maschinell potenzieren und trotzdem viele Homöopathika verkaufen!

Ein weiterer Aspekt ist die einwandfreie Konfektion und die Abgabe/der Versand der hergestellten Präparate. Dies sind Aspekte, die homöopathische Ärzte und Therapeuten beachten müssen - erst recht heute im Internet-Zeitalter, in dem

\section{KARGER}

Fax +4976145207 14
() 2014 S. Karger GmbH, Freiburg 
unzählige Anbieter im Netz mit verlockenden Angeboten auf sich aufmerksam machen.

\section{Abgabe durch wen?}

Weiter stellt sich die Frage, wer einem Patienten das verordnete homöopathische Medikament abgibt? Der Homöopath selber, eine Apotheke, mit der er zusammenarbeitet und $\mathrm{zu}$ der er den Patienten schickt, oder überlässt er es dem Patienten, sich das verordnete Homöopathikum zu beschaffen? Dabei stellen sich ungeahnt heikle Fragen!

Hahnemann hatte den Rat gegeben, homöopathische Medikamente selber herzustellen und auch selber abzugeben [1]. Er hatte zu den damaligen Apothekern zu wenig Vertrauen und argwöhnte, dass diese seine Herstellungsvorschriften nicht einhielten, weil sie darin keinen Sinn sahen.

In der Schweiz regulieren rechtliche Bestimmungen die Herstellung und die Abgabe von herkömmlichen und ebenso von homöopathischen und anthroposophischen Arzneimitteln.

Die Abgabe direkt durch den Homöopathen ist nur in den Kantonen gestattet, in denen die sogenannte Selbstdispensation rechtlich erlaubt ist. Nichtärztliche Therapeuten benötigen in diesen Kantonen eine Abgabebewilligung.

Eine andere Frage ist die Herstellung, für die es eigene gesetzliche Bestimmungen gibt.

\section{Bewilligung zur Herstellung}

Grundlage zur Herstellung von Arzneimitteln ist Artikel 5 des Heilmittelgesetzes (HMG), das für einen Betrieb ein entsprechendes Qualitätssicherungssystem voraussetzt.

Für die Herstellung homöopathischer und anthroposophischer Arzneimittel müssen neben den aner- kannten Regeln der «Guten Herstellungspraxis» (Good Manufacturing Practice (GMP)) [2] auch die anerkannten spezifischen Herstellungsvorschriften der homöopathischen und anthroposophischen Herstellungsverfahren eingehalten und ausreichend dokumentiert werden [3].

Die Ausgangsstoffe [4] müssen folgenden Kriterien entsprechen:

- den allgemeinen Anforderungen an Ausgangsstoffe der Pharmakopöe, des Homöopathischen Arzneibuches (HAB) [5], der Pharmacopé française [6] und der British Homoeopathic Pharmacopoeia [7],

- den für Homöopathika geltenden Monographien der Pharmakopöe und

- den jeweiligen Einzelmonographien des HAB, der Pharmacopé française, der Pharmakopöe oder, sofern dort keine Monographie vorhanden ist, den Einzelmonographien der HPUS (Homeopathic Pharmacopoeia of the United States) oder den entsprechenden Qualitätsmonographien der Hersteller.

Die schweizerische Gesetzgebung [8] unterscheidet zwischen homöopathischen Arzneien mit und ohne Indikationsangabe. Für beide Arten von Arzneien muss der Hersteller primär gemäss HMG aufgrund Erfüllung diverser Bedingungen und Anforderungen über eine Betriebsbewilligung [9] verfügen und GMPzertifiziert [2] sein.

\section{Registrierung und Abgabe}

Für homöopathische Arzneimittel mit Indikation muss ein vollständiges Zulassungsgesuch mit Unterlagen zu Qualität, Sicherheit und Wirksamkeit bei der Swissmedic eingereicht werden. Für die Zulassung eines homöopathischen Arzneimittels ohne Indikation ist eine vereinfachte Zulassung bzw. ein Meldeverfahren [10] vorgesehen.

Die Abgabekompetenzen werden insbesondere im Artikel 25 des
HMG geregelt. Besondere Komplexität kommt der Bestimmung zu, dass neben Personen, die verschreibungspflichtige Arzneimittel abgeben können (Ärzte, Apotheken), auch Drogerien und «Personen mit angemessener Ausbildung» Arzneimittel abgeben können.

Das Abfüllen von ein paar Globuli in ein kleines Abgabegefäss und das Auflösen von Globuli in Wasser oder Ethanol gelten bereits als Herstellungsschritte. Zur Herstellung homöopathischer Arzneien bedarf es aber einer Herstellungsbewilligung. Nur die allerwenigsten Praxen verfügen jedoch über eine solche.

\section{Einzeldosen}

Der homöopathischen Behandlungsweise entsprechend verordnet der Homöopath einem Patienten oft eine Einzeldosis eines homöopathischen Arzneimittels, z.B. 2 Globuli Arsenicum album C200, 2 Globuli Bryonia alba XM usw., die allenfalls nach einigen Wochen wiederholt wird. Dabei kommen die eben erwähnten rechtlichen Bestimmungen zur Anwendung. Verfügt der Homöopath über eine Abgabebewilligung, nimmt der Patient diese Einzeldosis meistens in der Praxis ein. Wie wird dies nun aber von Personen gehandhabt, die über keine Abgabebewilligung verfügen? In Kantonen ohne Selbstdispensation sind auch homöopathische Ärzte davon betroffen, die eine Einzeldosis verschreiben müssen. Welche Voraussetzungen müssen erfüllt sein, wenn der Homöopath dem Patienten eine Einzeldosis für eine Einnahme zu einem späteren Zeitpunkt mitgibt?

\section{Originalpackungen}

Die gängigen, in der Schweiz erhältlichen Originalpackungen von homöopathischen Einzelpotenzen enthalten eine grössere Anzahl Globuli, die eine Einzeldosis weit überschreiten. Es ist somit nicht sinnvoll, für eine Einzeldosis eine ganze Originalpackung abzugeben. Wie müssen 
Einzeldosen für die Mitgabe aber verpackt sein, damit alle gesetzlichen Bestimmungen erfüllt sind?

\section{Abgabe von Einzeldosen}

Bei der Nachfrage bei verschiedenen Kantonsapothekern wurde wiederholt auf ein Merkblatt der Dienststelle Gesundheit des Kantons Luzern verwiesen, das sehr ausführlich auf das Problem eingeht [11]. Darin sind die Vorschriften in Bezug auf die Verpackung und Beschriftung von Teilmengen genau geregelt und folgen dem Grundsatz: «Die Information auf und in der Originalpackung muss auch der Teilmenge mitgegeben werden. In jedem Fall muss die vollständige Rückverfolgbarkeit sichergestellt werden.»

Auf der Verpackung einer Teilmenge müssen sämtliche Angaben ersichtlich sein, wie es auch für die Originalpackung erforderlich ist, weiter der Patientenname und Jahrgang, der Name und die Adresse des Abgebers sowie das Abgabedatum.

In einem internen Schreiben der Schweizerischen Vereinigung homöopathischer Ärztinnen und Ärzte empfiehlt der Vorstand des SVHA seinen Mitgliedern, die das Recht auf Selbstdispensation besitzen, die Praxisapotheke mit konfektionierten Originalpackungen von Einzeldosen auszurüsten.

\section{Einzeldosen im Handel}

Die in der Schweiz ansässigen Homöopathiefirmen bieten verschiedene Packungsgrössen an, die dem Anliegen der Einzeldosen mehr oder weniger entgegenkommen.
Verschiedene Westschweizer Firmen wie die Laboratoire D. SchmidtNagel SA, die Boiron SA oder die Pharmacie M. Ernest Gamma haben in ihrem Sortiment sogenannte 1-Dosis-Packungen. Dabei handelt es sich um Röhrchen, die 1 Gramm, d.h. 300 sehr kleine Globuli, enthalten, die als Einzeldosen gedacht sind. Wer aber schon einmal eine solche Einzeldosis eingenommen hat, weiss, dass es recht lange dauert, bis die Globuli im Mund zergangen sind. Ausserdem übersteigen diese 300 Globuli mengenmässig eine Einzeldosis bei Weitem.

Die in Küssnacht ansässige OMIDA AG bietet viele ihrer homöopathischen Potenzen in Röhrchen à $2 \mathrm{Gramm}$ an. Diese enthalten ca. 200 Globuli, die erheblich grösser sind als die Globuli der einzelnen Westschweizer Dosis. Allerdings besteht auch hier das eben geschilderte Problem. Wenn Homöopathen aus diesen 1- bzw. 2-Gramm-Röhrchen mehrere Einzeldosen machen, dann müssen sie den Aufwand eines gesetzeskonformen Dispensationsgefässes treiben, was viel Zeit in Anspruch nimmt.

\section{Easyclick}

Erstaunlicherweise bietet nur ein Hersteller homöopathischer Einzelpotenzen, die Spagyros SA in Gümligen, echte Einzeldosen an. Dabei handelt es sich um Easyclick genannte Packungen mit 5 Globuli, die als Einzeldosis eingenommen werden. Einmal geöffnet, können die Easyclick nicht mehr verschlossen und müssen entsorgt werden.

\section{Fazit}

Für die Herstellung homöopathischer Arzneimittel gibt es klare gesetzliche Vorschriften, die garantieren, dass diese nach GMP und den Regeln homöopathischer Herstellung produziert werden. Ärztliche Homöopathen und Heilpraktiker sind gut beraten, ausschliesslich homöopathische Potenzen, je nach Kanton und Bewilligung, entweder selber abzugeben oder zu verschreiben/zu empfehlen, die von einer solchen Firma hergestellt werden.

Bei der Abgabe/Verordnung von Einzeldosen müssen die gesetzlichen Bestimmungen ebenfalls eingehalten werden. Das Easyclick-System der Firma Spagyros SA ist das einzige in der Schweiz erhältliche EinzeldosisSystem, das die Abgabe von Einzeldosen ideal regelt.

\section{Literatur}

1 Hahnemann S: Organon VI, \$264 und 265.

2 GMP: «Good Manufacuring Practice» - Verordnung über die Bewilligungen im Arzneimittelbereich (AMBV), Art. 3-5 und Anhang 1.

3 Art. 13 KPAV (Komplementär- und Phytoarzneimittel-Verordnung).

4 Art. $8 \mathrm{KPAV}$.

5 Homöopathisches Arzneibuch, zu grossen Teilen überführt in die Pharmacopoea Europaea (Ph. Eur.).

6 Pharmacopé française.

7 British homoeopathic pharmacopoeia.

8 Heilmittelgesetz (HMG).

9 HMG Art. 5-7.

10 Art. $19 \mathrm{KPAV}$.

11 Kanton Luzern, Dienststelle Gesundheit, $\mathrm{MB} /$ Abgabe von Teilmengen aus Originalpackungen, V01 300911 spi. 